\title{
PATENT DUCTUS ARTERIOSUS WITH PULMONARY HYPERTENSION AND REVERSED SHUNT
}

\author{
BY \\ GEORGE SMITH \\ From the Department of Surgery, University of Glasgow, and the Western Infirmary, Glasgow
}

Received November 11, 1953

Patency of the ductus arteriosus usually allows entry of blood from the aorta into the lowpressure pulmonary circulation and is associated with a fairly constant clinical picture. In recent years atypical cases have been reported, in which one or more of the usual clinical features have been missing, most commonly the continuous murmur. Investigations made on such cases during life have shown that there was pulmonary hypertension associated with patency of the ductus arteriosus (Dushane and Montgomery, 1948; Myers et al., 1951); in others, raised pressure in the pulmonary circuit was inferred merely from autopsy findings (Chapman and Robbins, 1944; Ulrich, 1947).

Gross pulmonary hypertension associated with patent ductus and cyanosis of the patient has been reported by Douglas et al. (1947), Pritchard et al. (1950), and Campbell and Hudson (1950, 1951). In only three reports, however, are physiological data complete enough to substantiate a right to left shunt through the ductus to account for this cyanosis (Johnson et al., 1950; Dammann et al., 1953; and Hultgren et al. 1953).

In the present case cyanosis was present from birth, a machinery murmur was never heard, gross pulmonary hypertension was present, and clear proof was obtained during life that blood flowed through the ductus from the pulmonary artery to the aorta. The purpose of this account is to emphasize the significance of this syndrome and to stress the inadvisability of operative closure of the ductus.

\section{CASE REPORT}

The patient, aged 19 years, was born of a full-time normal pregnancy. From birth it was noted that her colour was bluish and this continued; she started at a special school at the age of 6 years. Throughout her life she was breathless on even slight exertion, but this became worse at the age of 17 years. Also on exertion from the age of 13 years she felt a tightness in the chest over the præcordial area. If she rested, these symptoms passed off rapidly, but if she forced herself to carry on in spite of them, she became bluish in colour. She always felt tired.

There was no history of pulmonary infections or rheumatic attacks. She had scarlet fever at 2 years, mastoiditis at 17 years, and convulsions up to the age of 12 years. None of her eight siblings had apparent congenital defects.

The patient was poorly developed, of gracile habitus, and weighed $90 \mathrm{lb}$; there was no scoliosis. Slight cyanosis of the lips, fingers, and toes was present and there was fullness of the nail beds of the fingers and early clubbing of the toes. No difference in the degree of cyanosis at these sites was noted. She had a well marked malar flush of the type often associated with mitral stenosis. Blood pressure was 95/60 in the arms and there were pulsations in both femoral arteries. The pulse rate was 60 a minute, regular in time and force. The heart was not enlarged. No thrill was felt. The second heart sound was split and accentuated at the pulmonic area. There was a blowing murmur throughout systole heard down the left sternal border and maximal at the inner end of the third left interspace. The lungs were clear. Red blood count was 4.5 millions per cu. mm., hæmoglobin $13.8 \mathrm{~g}$. and hæmatocrit 45 per cent.

A phonocardiogram showed a systolic murmur and a very short early diastolic murmur at the base, but four days later a systolic murmur only. The examination was carried out with the patient at rest, after 
exercise and lying on her left side. Electrocardiograms showed right axis deviation and right ventricular preponderance (Fig. 1).

X-ray and fluoroscopic examination confirmed that the heart was not enlarged in its transverse diameter and the cardiothoracic ratio was 45 per cent (Fig. 2). The pulmonary conus was dilated and showed expansile pulsation. Pulmonary arteries were present on both sides. The lung fields were vascularized within normal limits. The right ventricle appeared to be enlarged.

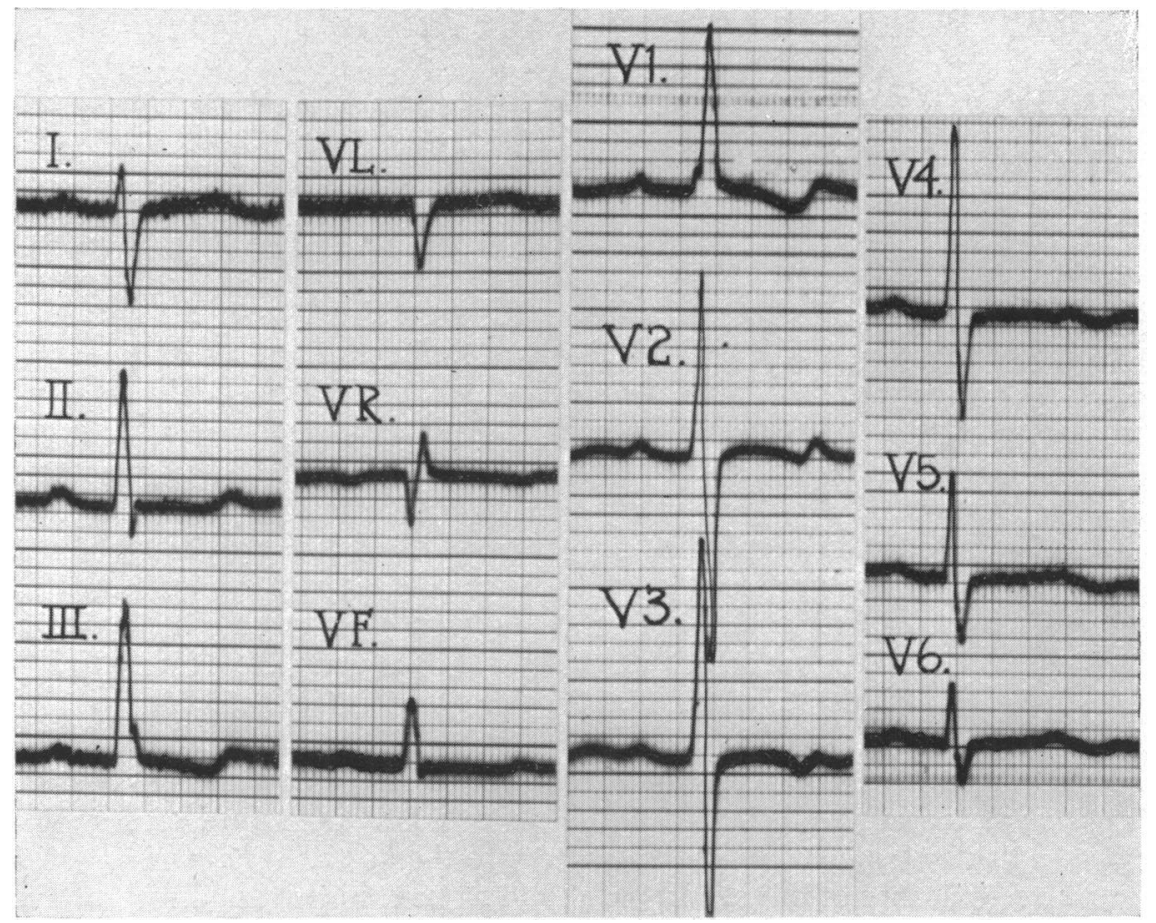

Fig. 1.-Electrocardiogram showing right axis deviation and right ventricular preponderance.

At cardiac catheterization the left main pulmonary artery was entered. On withdrawing the catheter slightly and again advancing, the aorta was entered and the catheter passed down to the upper abdominal aorta. The data obtained from this procedure were as follows:

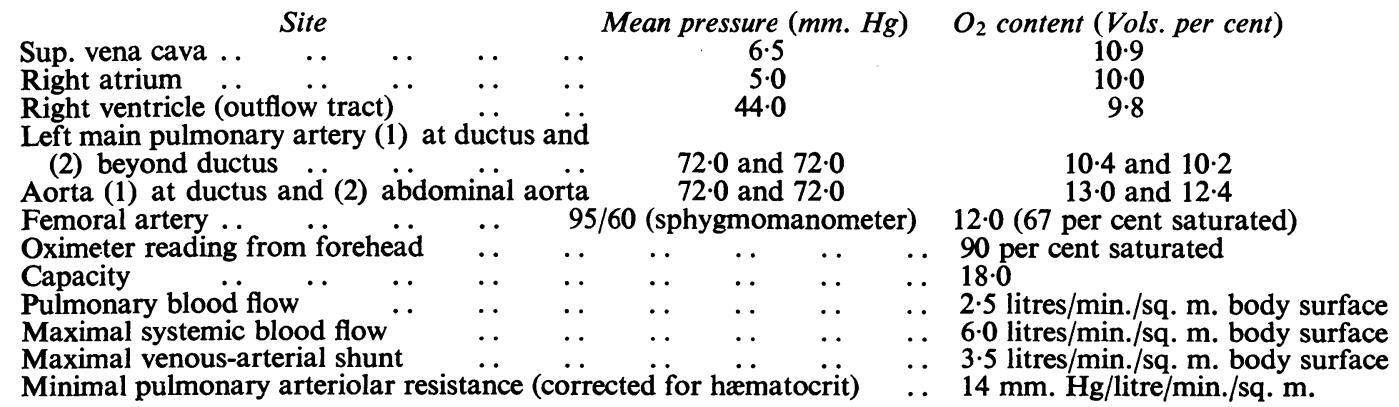

These findings were interpreted as indicative of a patent ductus arteriosus situated at the common site, the aortic opening being just distal to the left subclavian artery. The pulmonary artery pressure was such that blood was apparently passing into the aorta causing the lowered oxygen content in the blood to the lower part of the body.

After rest in bed for a week, the patient's colour appeared to improve and in addition to the systolic murmur a soft early diastolic one became audible at the inner end of the left third intercostal space. 


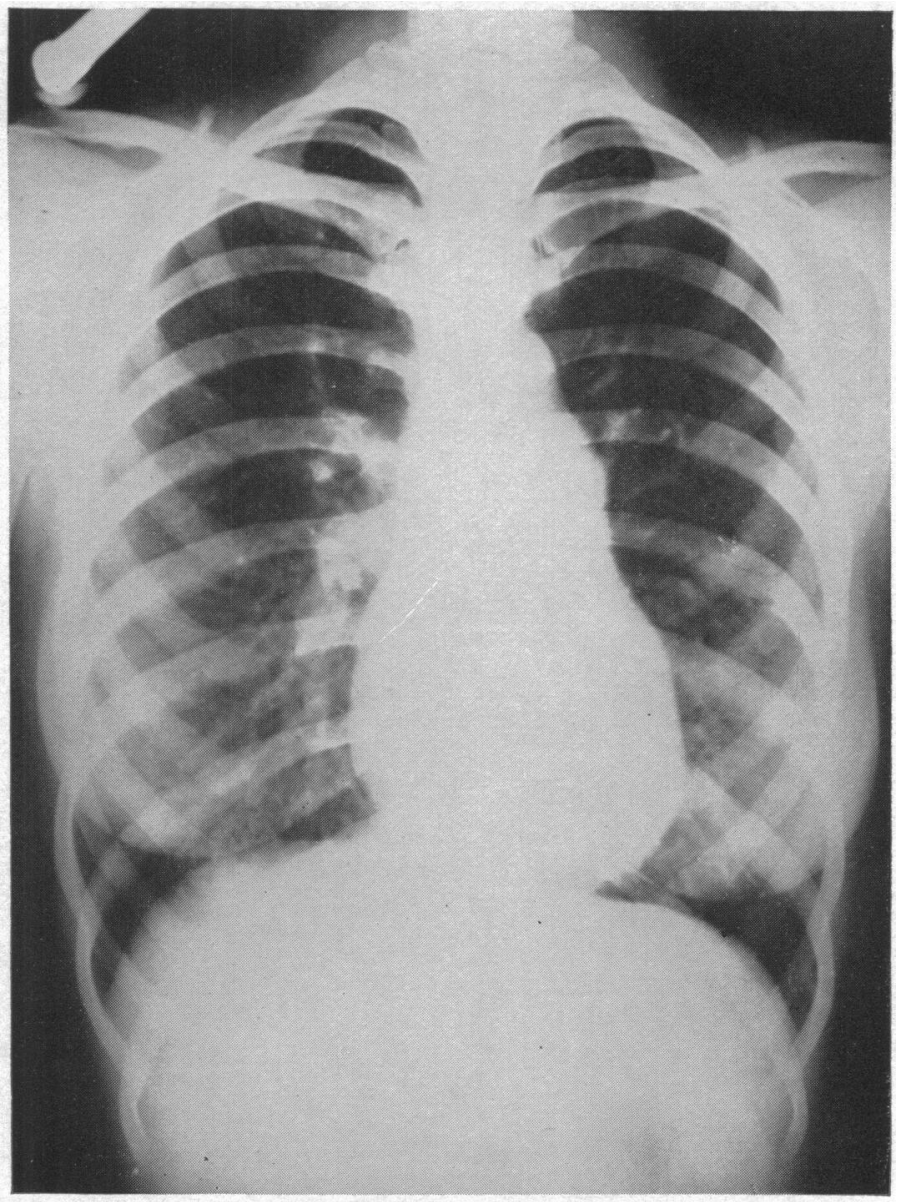

FIG. 2.-Teleradiogram of the heart. There is prominence of the main and right pulmonary arteries.

Left lateral thoracotomy was performed by Mr. W. A. Mackey. The pulmonary artery was $35 \mathrm{~mm}$. external diameter while the aorta at the arch was $25 \mathrm{~mm}$. The descending portion of this latter vessel was $20 \mathrm{~mm}$. in external diameter. There was a large ductus present at the usual site. The duct was short and about $7.5 \mathrm{~mm}$. in its outside diameter. The pulmonary artery mear pressure was $89.5 \mathrm{~mm}$. while that in the aorta was $88.0 \mathrm{~mm}$. Hg. Temporary occlusion of the ductus was carried out for a period of 15 minutes, during which time the mean pressure in the pulmonary artery fell to $54.5 \mathrm{~mm}$., while the mean aortic pressure was $80 \mathrm{~mm}$. This was interpreted as indicating that the vascular changes in the pulmonary bed were probably not fixed. The ductus was then permanently ligated. There was no thrill in the ductus area before or after ligation. The tip of the lingula of the left lung was removed for histological examination.

She recovered satisfactorily from the anæsthetic but about 9 hours later became restless, then rapidly comatose. Auricular fibrillation developed and shortly thereafter death occurred.

Post-mortem Examination. This was carried out 36 hours later (Dr. J. A. Milne). The thoracic organs were removed en masse. The lungs were moderately inflated with bellows and the pulmonary artery perfused with normal saline. Then a warm barium gelatine emulsion was run into the pulmonary artery at a pressure of $100 \mathrm{~cm}$. of water. When the gelatine was set the lungs were X-rayed. The specimen was then fixed in 5 per cent formalin and examined in detail after a few days.

The pleural surfaces were normal and there was no gross disease of the lung parenchyma. The 
pulmonary arteries were dilated and sclerotic but no abnormalities were found in the pulmonary veins. The bronchial tree appeared normal.

The right ventricle, though somewhat dilated, was hypertrophied, the wall measuring $10 \mathrm{~mm}$. in thickness while that of the left ventricle was $15 \mathrm{~mm}$. thick (Fig. 3). The atria appeared normal. No abnormal intracardiac communication existed. The heart valves, the endocardium, the heart muscle, the epicardium, and the coronary arterial tree showed no abnormality.

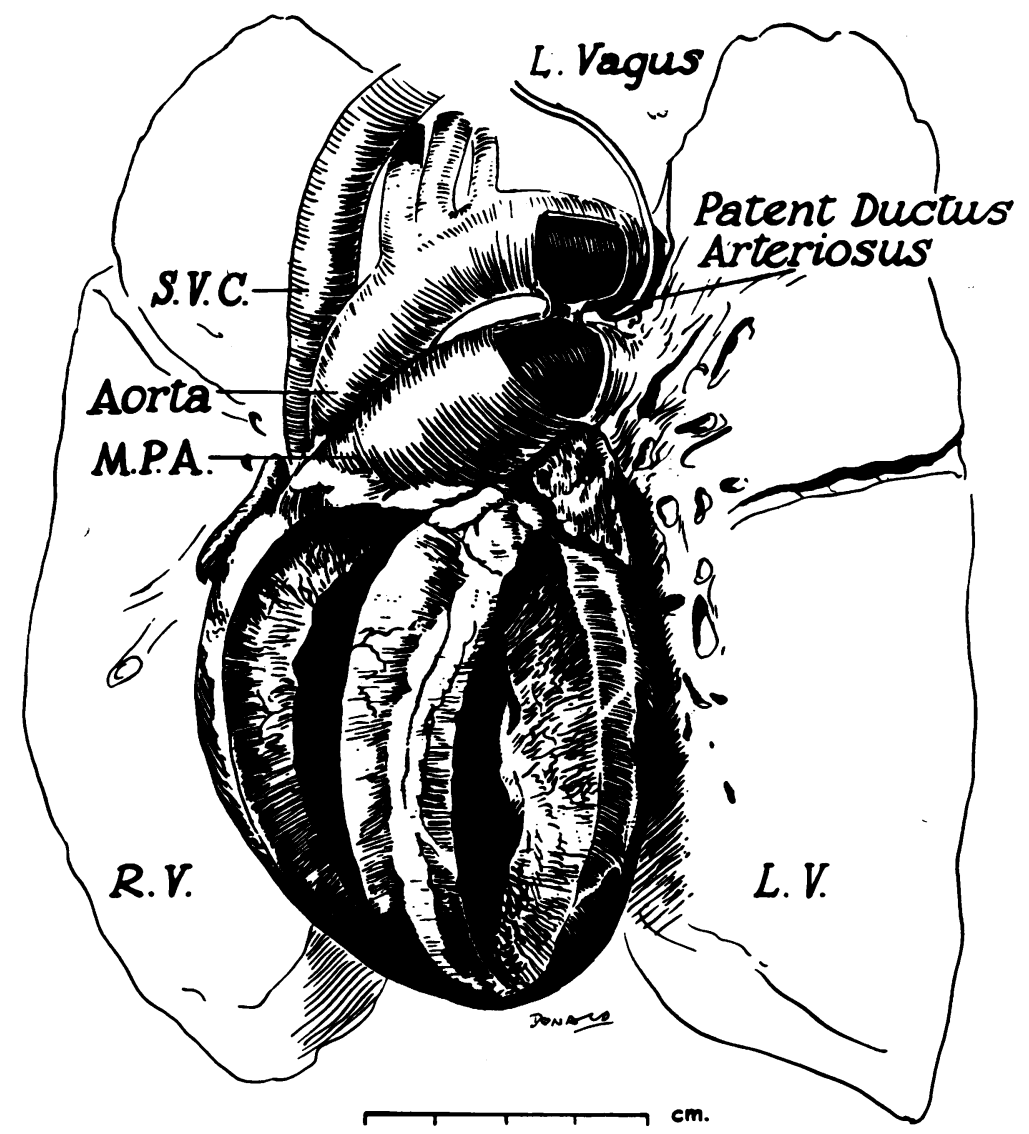

Fig. 3.-Sketch to illustrate the relative size of the pulmonary artery and the aorta. The right ventricular wall is thickened.

The main pulmonary artery measured $30 \mathrm{~mm}$. in diameter while the ascending aorta was $20 \mathrm{~mm}$. Between the left main pulmonary artery at its origin and that part of the aortic arch just distal to the origin of the left subclavian artery there was a short ductus completely occluded by surgical ligatures. Just beyond the ductus the aorta measured only $15 \mathrm{~mm}$. but there was no localized constriction in the vessel.

At both the aortic and pulmonary ends of the ductus there were a few small scattered plaques of atheroma but no " jet" lesion of the intima of either of these vessels existed in the ductus area. The intima of the aorta and the pulmonary artery together with its larger branches were free of macroscopic atheromatous change.

No significant abnormality was found in the rest of the body.

The lung biopsy and tissue from all lobes removed post mortem were examined microscopically and all showed the same lesions. The alveolar walls, capillaries, and veins appeared normal. 
In the larger elastic pulmonary arteries there was medial thickening with early intimal atheromatous change. The larger muscular arteries showed no apparent abnormality. The medium-sized ones were the site of intimal thickening with subintimal fibrosis and reduplication of the internal elastic lamina while the smaller muscular arteries showed these changes to a relatively greater extent (Fig. 4 and 5). In the arterioles, the intimal thickening in places almost occluded the lumen.

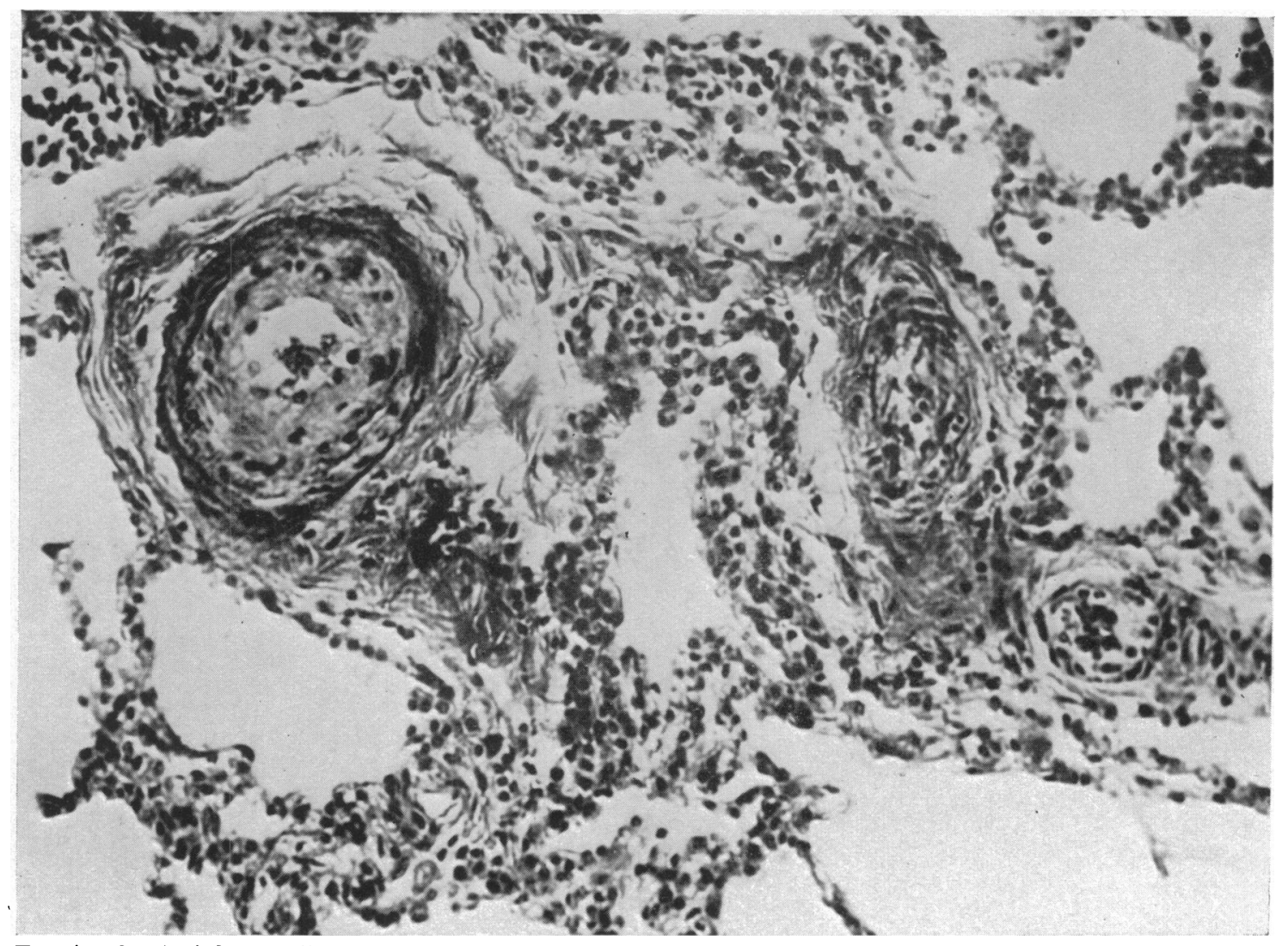

Fig. 4. - On the left a small muscular pulmonary artery shows marked subintimal fibrosis; the arteriole to the right of the field shows thickening of its wall. (Haematoxylin and eosin, $\times 200$.)

A most striking feature was the presence of thrombosis of the medium and smaller arteries often at their origin from a larger branch (Fig. 6). All stages from recent occlusion to reorganization of these thrombi existed. In the proximity of some of these vessels thin-walled, endothelial-lined blood spaces were seen. These resembled angiomata of the cavernous type, but their significance is uncertain.

In the area of the ductus, the walls of the ductus and its pulmonary artery end showed early atheromatous degeneration with the endothelial covering still intact. No thrombi were seen.

\section{Discussion}

The evidence in the present case seems adequate to substantiate a right to left shunt through the ductus. The difference in oxygen saturation between the blood to the head and that to the lower part of the body indicates admixture of venous blood with that in the aorta at some point distal to the origin of the vessels to the head, and the only communication found at this site was a patent ductus arteriosus. The pressures in the pulmonary artery both at catheterization and at operation are such that blood could flow from the pulmonary artery into the aorta. 


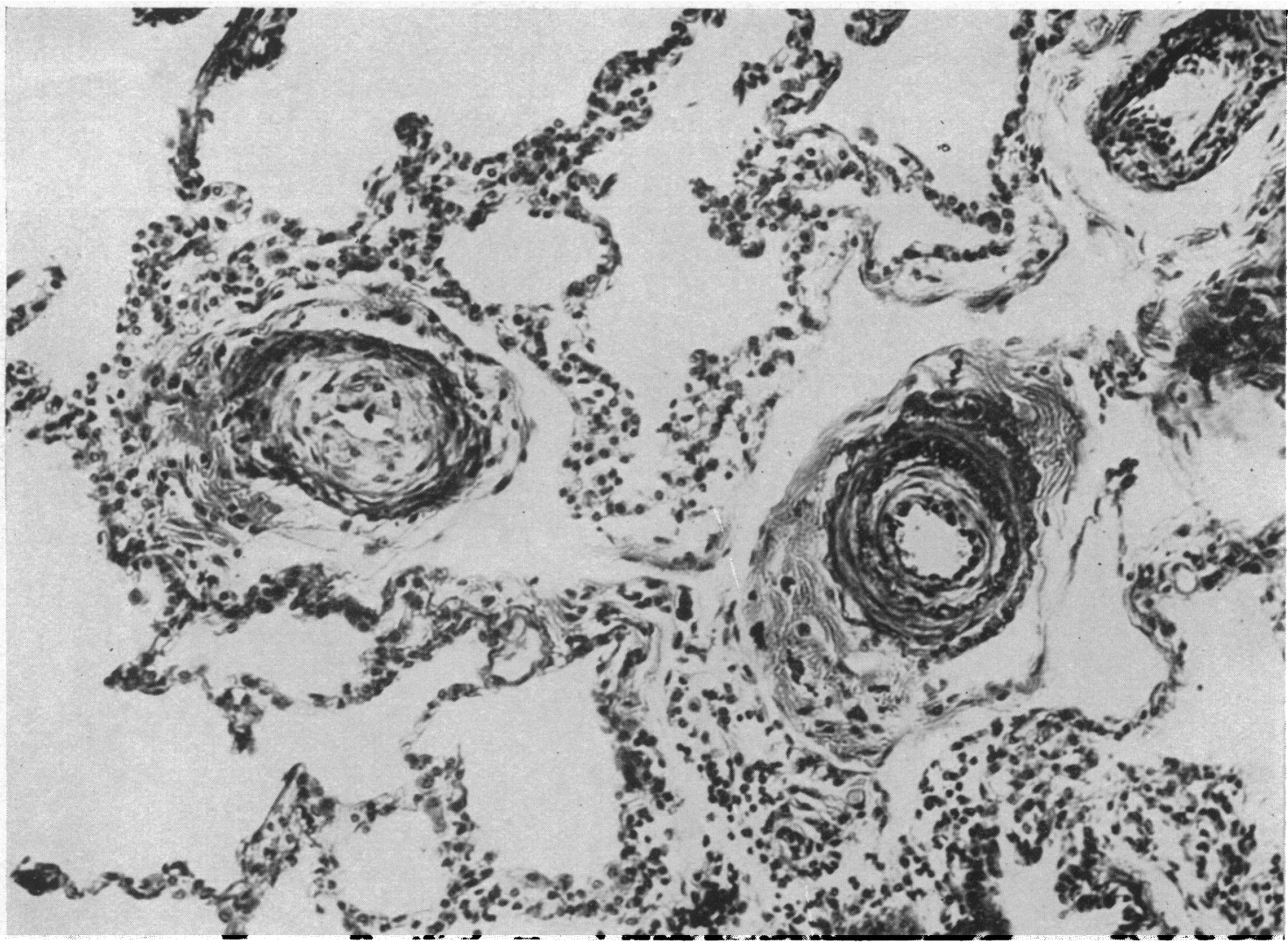

FIG. 5.-Section to show smaller muscular pulmonary arteries. There is an increase of elastica and subintimal fibrosis. (Weigert, $\times 200$.)

Calculation of the minimal pulmonary arteriolar resistance (assuming a maximal pulmonary vein mean pressure of $35 \mathrm{~mm}$.) shows that this is three times the value obtained in the usual type of ductus case. Since there is neither gross nor microscopic evidence that the pulmonary vein mean pressure should have been other than normal, the pulmonary arteriolar resistance may have been at least seven times greater than that found in the usual case of the patent ductus arteriosus.

Further evidence of increased resistance in the arterial bed is afforded by the X-ray examination of the injected lungs and by the state of the arteries and arterioles on histological examination. Instead of the normal branching pattern, the injection mass stops abruptly in arteries corresponding in size to these museular arteries seen to be the site of thrombosis in the histological sections (Fig. 6). This occlusive process is widespread. The histological evidence of hyperplastic arteriolar sclerosis is probably of greater significance.

As to the cause of the pulmonary hypertension, four possibilities exist.

(1) In the beginning the case may have been merely one of patent ductus and the pulmonary hypertension may have resulted subsequently from prolonged increase in the pulmonary blood flow. The onset of symptoms early in life together with persistent cyanosis from birth make this improbable. Furthermore, the changes seen in the vessels are quite different from these in the usual type of patent ductus even where this channel is large and the pulmonary blood flow great. Thus Welch and Kinney (1948), found that the pulmonary arterial tree did not differ from that in normal controls in 24 of 25 cases of large patent ductus: it is significant that the exception was the case described previously by Chapman and Robbins in which pulmonary hypertension was present.

(2) The second possibility is repeated thrombosis or embolism in the smaller pulmonary arteries. 


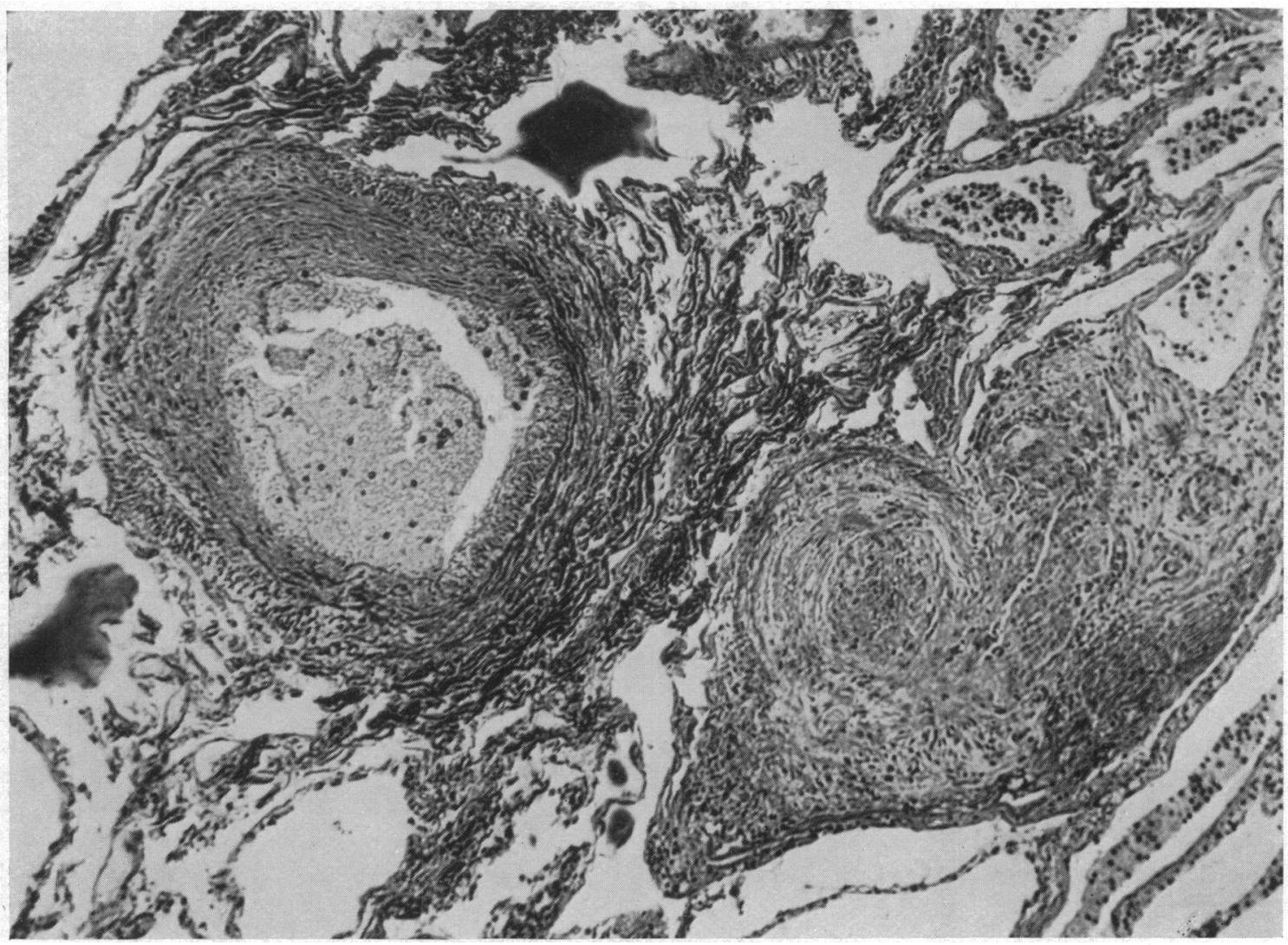

Fig. 6.-Section of medium-sized pulmonary artery showing medial hypertrophy and recent thrombus in the vessels to the right of the field. (Haematoxylin and eosin, $\times 100$.)

Since the site of occlusion is frequently at a point where a smaller artery branches off a larger trunk embolism seems more likely. In the absence, however, of endarteritis, endocarditis, polycythæmia, and a failing heart, the source of such emboli is unknown; eddy currents in the vicinity of the ductus may have contributed to their formation. Whatever the origin of these thrombi, it is considered that they are secondary phenomena in an already existing pulmonary hypertensive state, although it is known that multiple pulmonary emboli can produce pulmonary hypertension (Castleman and Bland, 1946). It appears certain that such a process contributed to the degree of pulmonary hypertension by gradually diminishing the cross-sectional area of the pulmonary arterial bed. Further, there is ample histological evidence that the process was a continuing one.

(3) Continuing attacks of pulmonary arteritis is the third possible cause for the pulmonary hypertension. While these occur in some cases with pulmonary hypertension as distinct from the "arteritis" set up by lodging of emboli in the pulmonary arterial tree, the very early onset of symptoms in the present case render this most improbable.

(4) The fourth possibility is that some congenital or neonatal mechanism exists whereby the equal pressures in the fotal systemic and pulmonary circulation persist into postnatal life. The organic changes of pulmonary hypertension would then follow. This thesis appears the most attractive in the present case. Further, since the vascular changes are progressive such a hypothesis can be applied to these cases of reversal of flow in the ductus where the onset of cyanosis occurs, not at birth, but later in life.

If such a cause for the pulmonary hypertension be accepted, operation must be withheld where 
there is evidence of reversal of flow through a ductus arteriosus in the light of the present case and Case 2 of Dammann et al. The ductus is persisting because of the congenital pulmonary hypertension, and serving as a safety valve for the right heart. This is in contrast to the cases of pulmonary hypertension with patent ductus but without evidence of right to left shunt. In these the ductus can be closed without immediate mortality, and such closure may be beneficial in the long run by diminishing the pulmonary blood flow, but further long-term observation of operated cases is needed to prove this.

The slight fall in mean aortic pressure on occlusion of the ductus can be explained on the basis of less blood passing into the aorta. The marked fall in pulmonary artery mean pressure must have resulted from two factors. Either the output of the right heart became less from the sudden extra load thrown on it or the vascular resistance in the pulmonary bed decreased. The former is more likely in view of the patient's death nine hours after closure of the ductus and the pathological findings. It may be that both factors played a part.

\section{SUMMARY}

A case of pulmonary hypertension with patent ductus arteriosus is presented. It is believed that the pulmonary hypertension was congenital in origin.

The murmurs were atypical and the patient showed intermittent cyanosis from birth. It is demonstrated that reversal of flow through the ductus occurred. Closure of the ductus in such cases may be fatal.

I wish to thank Dr. J. D. O. Kerr for the electrocardiographic and phonccardiographic records.

\section{REFERENCES}

Campbell, M., and Hudson, R. (1951). Guy's Hosp. Rep., 100, 26.

(1952). Guy's Hosp. Rep., 101, 32.

Castleman, B., and Bland, E. F. (1946). Arch. Path., 42, 581.

Chapman, C., and Robbins, S. (1944). Ann. intern. Med., 21, 312.

Dammann, J. F., Berthrong, M., and Bing, R. J. (1953). Bull. Johns Hopkins Hosp., 92, 128.

Douglas, J., Burchell, H., Edwards, J., Dry, T., and Parker, R. (1947). Proc. Staff Meet. Mayo Clin., 22, 413.

Dushane, J., and Montgomery, G. (1948). Proc. Staff Meet. Mayo Clin., 23, 305.

Hultgren, H., Selzer, A., Purdy, A., Holman, E., and Gerbode, F. (1953). Circulation, 8, 15.

Johnson, R., Wermer, P., Kuschner, M., and Cournand, A. (1950). Circulation, 1, 1293.

Welch, K. J., and Kinney, T. D. (1948). Amer. J. Path., 24, 729.

Myers, G., Scannell, J., Wyman, S., Dimond, E., and Hurst, J. (1951). Amer. Heart J., 41, 819.

Pritchard, W., Brofman, B., and Hellerstein, H. (1950). J. Lab. Clin. Med., 36, 974.

Ulrich, H. (1947). Acta med. Scand., 196, 160. 